\title{
Parental Rejection, Personality, and Depression in the Prediction of Suicidality in a Sample of Nonclinical Young Adults
}

\author{
Ana Teresa Sobrinho, MSc, \\ and Rui C. Campos, PhD \\ University of Évora
}

\author{
Ronald R. Holden, PhD \\ Queen's University
}

This study tested a prediction model of suicidality in a sample of young adults. Predictor variables included perceived parental rejection, self-criticism, neediness, and depression. Participants $(N=165)$ responded to the Depressive Experiences Questionnaire, the Inventory for Assessing Memories of Parental Rearing Behavior, the Center for Epidemiological Studies Depression Scale, and the Suicide Behaviors Questionnaire-Revised. Perceived parental rejection, personality, and depression were assessed initially at Time 1 , and depression again and suicidality were assessed 5 months later at Time 2. The proposed structural equation model fit the observed data well in a sample of young adults. Parental rejection demonstrated direct and indirect relationships with suicidality, and self-criticism and neediness each had indirect associations with suicidality. Depression was directly related to suicidality. Implications for clinical practice are discussed.

Keywords: parental rejection, self-criticism, neediness, depression, suicidality

Suicide is one of the primary causes of death in the world, and as such, is considered a major public health problem. Globally, it is the cause of nearly 1 million deaths annually, and this number continues to increase. In 1998, death by suicide represented $1.8 \%$ of the mortality rate, with an expectation that the rate will increase to $2.4 \%$ by 2020 (World Health Organization, 2012). Furthermore, suicide attempts are estimated to have a rate 10 to 20 times higher than deaths by suicide (Fotti, Katz, Afifi, \& Cox, 2006). Of particular note, rates of suicidal ideation and suicidal behavior increase toward the end of adoles-

This article was published Online First January 11, 2016.

Ana Teresa Sobrinho, MSc, Department of Psychology, University of Évora; Rui C. Campos, $\mathrm{PhD}$, Department of Psychology and Research Center on Education and Psychology, University of Évora; Ronald R. Holden, PhD, Department of Psychology, Queen's University.

We would like to acknowledge the contributions of all of the participants in this study.

Correspondence concerning this article should be addressed to Rui C. Campos, PhD, Departmento of Psicologia, Escola de Ciências Sociais, Universidade de Évora, Apartado 94, Évora, Portugal 7002-554. E-mail: rcampos@uevora.pt 